\title{
el pavimento pretensado del Centro de Explotación de la Blécherette
}

\author{
Mont-sur-Lausanne * SUIZA
}

$514-66$

\section{sinopsis}

La realización de este centro de la Blécherette, que comprende unos $10.500 \mathrm{~m}^{2}$ de losas sobre pilares, utilizadas como plataforma de circulación para automoviles, ha permitidó la colosado que presenta las siguientes características: consigue, por sí misma, la estanquidad; soporta las cargas usuales para los puentes de vehículos, y sufre deformaciones limitadas, a pesar de un desencofrado rápido.

Si los problemas de ejecución llevaron sobre todo al estudio y elección de los hormigones, asi como al establecimiento de un programa cálculo, por su parte, hubo de tener en cuenta las especiales características de las sobrecargas que excluían, de entrada, la utilización de métodos empíricos.

Los cálculos se hìcieron con una cierta analogía respecto a los de un puente de carretera, pero con todos los elementos bidireccionales.

Gracias al ordenador y después de alguna experiencia, el dimensionamiento del pretensado y la verificación de los esfuerzos de flexión se abardarse yordarse el control al punzonamiento de la losa campo muy complejo en donde, a falta de investigaciones más profundas o de ensayos sobre modelo, los métodos tradicionales hubieron de ser adaptados con intuición y lógica.

\section{INTRODUCCION}

Este gran complejo, que ocupa un terreno de $56.000 \mathrm{~m}^{2}$ en el Municipio de Mont-sur-Lausanne, junto a la desviación Blécherette de la Autopista núm. 9, está constituido por los centros de la policía de tráfico y de conservación de carreteras, y por la oficina de pesos y medidas.

Su disposición general responde, principalmente, a una utilización racional del terreno y de su topografía. Muestra de ello es la entrada principal del centro que, dispuesta al mismo nivel que el camino de Lanterne, se prolonga en una plataforma de circulación que sirve de techo para un piso bajo, en el que están situados el garaje policial y los depósitos. Al norte de la parcela se encuentra el aparcamiento general, realizado en dos niveles.

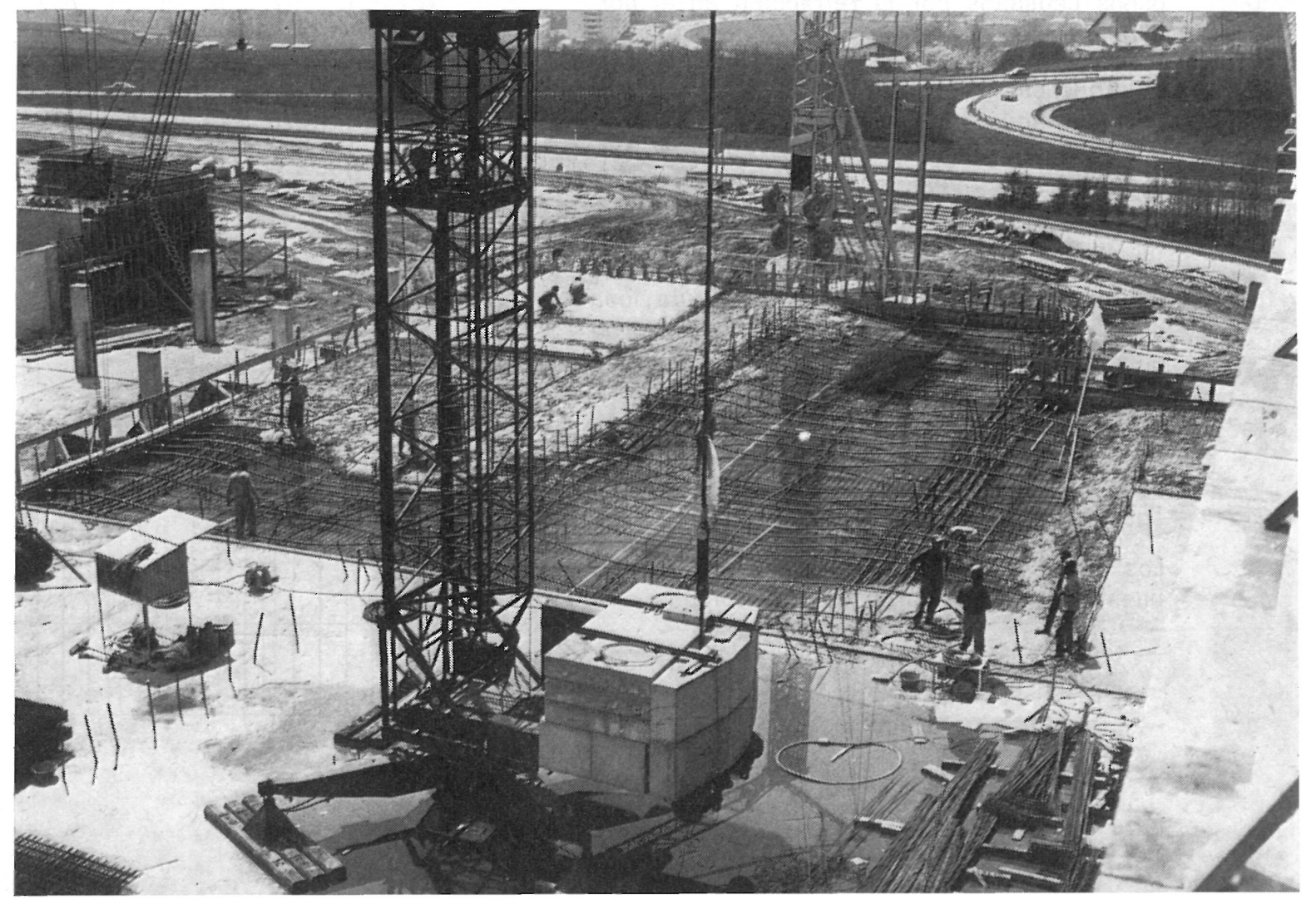




\section{CONSIDERACIONES GENERALES SOBRE LAS LOSAS GRUESAS}

La estructura de las plataformas de circulación debía satisfacer dos condiciones esenciales: servir de cubierta al garaje, aparcamiento y depósitos; y dar acceso vehicular a los diversos edificios del centro, para lo cual debía tener la suficiente resistencia para soportar las sobrecargas normales de un puente y resistir los efectos térmicos de una construcción al aire libre.

La segunda condición condujo a elegir losas de gran espesor y planas, ya que los gálibos necesarios de altura libre impedían la utilización de dinteles.

La técnica convencional confía el papel sustentante al hormigón armado, la estanquidad a una delgada capa de asfalto o mástic bituminoso, y la protección de esta última a un revestimiento hidrocarbonado cuya capa superior o de desgaste absorbe el tráfico.

En este caso, y desde los primeros estudios, se trató de encontrar una solución integral a base de hormigón, con la eventualidad de añadir una capa de desgaste bituminosa.

Por todo lo anterior resultó lógico el empleo de losas pretensadas para el pavimento. Estas tienen además la ventaja de que al mantener comprimido al hormigón bajo carga permanente limita sus tracciones bajo carga de servicio; y por otra parte, mediante una puesta en tensión parcial se pueden prevenir los daños causados por la retracción en el hormigón fresco.

La utilización del pretensado no introduce ninguna nueva limitación en lo que concierne a los apoyos de la estructura; de todas formas éstos se dispusieron móviles, mediante bloques de neopreno, para prevenir los efectos térmicos.

En total se construyeron $10.550 \mathrm{~m}^{2}$ de losa distribuidos de la siguiente manera: 3.700 en el garaje de la policía; 4.950 en los depósitos, y 1.900 en el aparcamiento. Toda esta superficie se proyectó con un sistema de pendientes y puntos bajos con rejillas de recogida, para asegurar una buena eliminación de las aguas de lluvia.

Los estudios del tipo de construcción propuesto se desarrollaron en tres campos:

- tecnología del hormigón;

- cálculo de la estructura; y

- problemas de ejecución.
A Diagrama de My, columna 12 , Cas $N{ }^{\circ} 5$

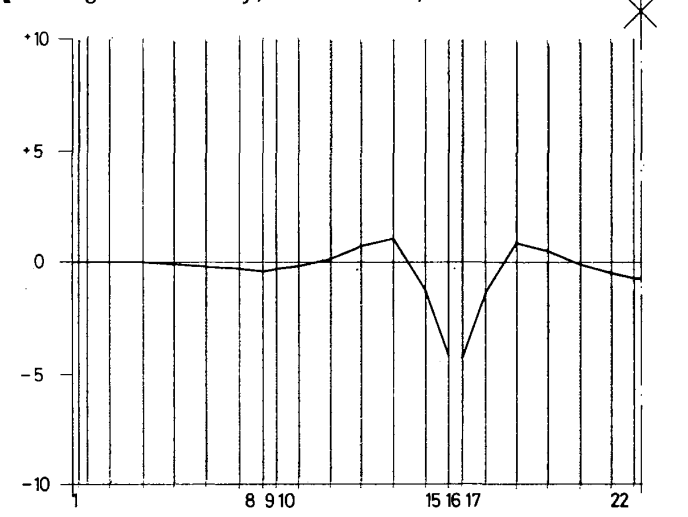

B Diagrama de My, columna 8 , Cas $\mathrm{N} \cdot 5$

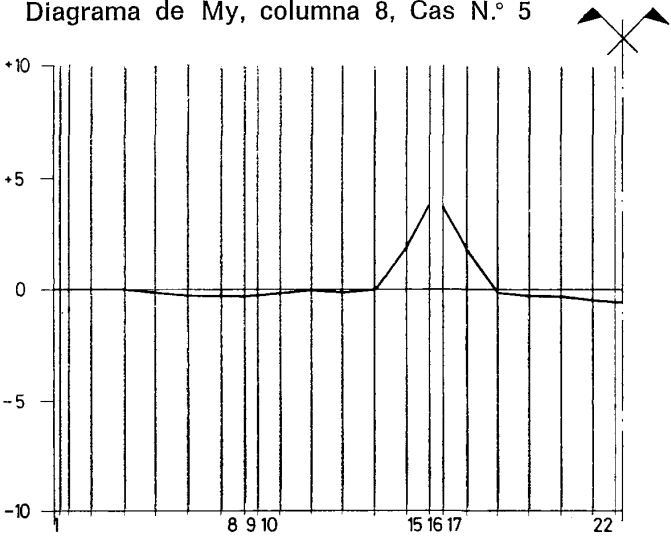

C Variación de $\mathrm{Mx}$, columna 12, Cas N. 5

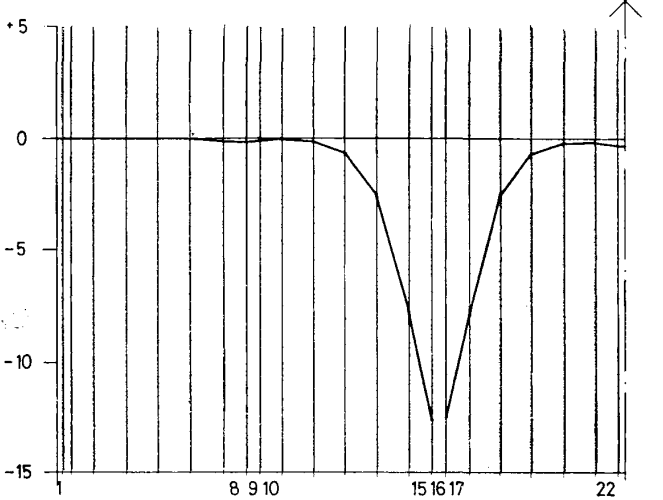

D Variación de $\mathrm{Mx}$, columna 8, Cas $\mathrm{N} .^{\circ} 5$

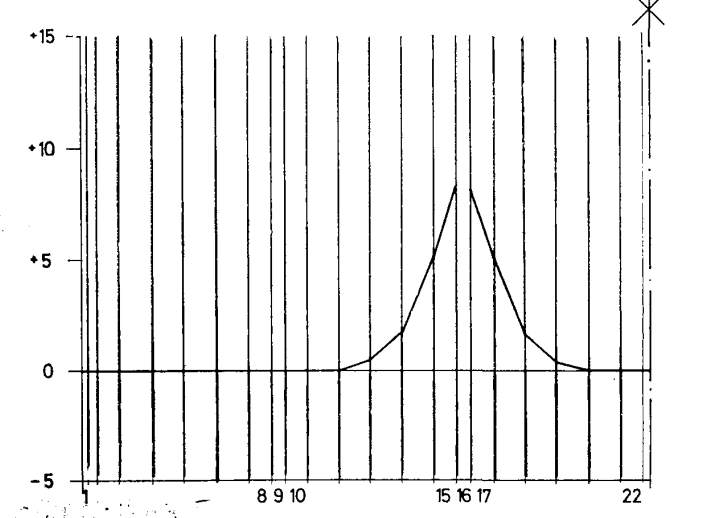

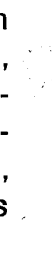




\section{ELECCION DE LOS HORMIGONES}

La naturaleza de la obra, relativamente gruesa dado el espesor de $50 \mathrm{~cm}$ de la losa, hacía aparecer el peligro de la retracción, tanto en el hormigón fresco como en el endurecido. Para minimizar este problema, el hormigón, al colocarlo, debía presentar unos valores limitados de dosificación, retracción y desprendimiento de calor, para una resistencia y compacidad normales.

La resistencia a la retracción del mortero es mejor si los agregados forman un esqueleto rígido, lo que se consigue aumentando el tamaño de los granos. En este caso, y gracias al pretensado que tiene una pequeña densidad de armaduras, se pudo aumentar el tamaño máximo de los granos hasta $60 \mathrm{~mm}$, con lo que la dosificación de cemento disminuye a $260 \mathrm{~kg} / \mathrm{m}^{3}$, sin modificación de la resistencia mecánica respecto al hormigón BH-300 de granulometría 0-30 mm (Teoría de Faury).

El uso de un aditivo no era, en principio, indispensable, pero, convenientemente elegido, podía mejorar la docilidad y evitar la segregación.

Se empleó un distribuidor de aire por presentar las ventajas de: tapar los capilares por la formación de meniscos; conservar la higrometría del medio; y preservar al hormigón del gel.

Después de numerosos ensayos efectuados con la colaboración del Prof. Daxelhofer y el Laboratorio de Materiales Pétreos, para su puesta a punto, se escogió un hormigón de granulometría discontinua 0-3, 8-15 y 30-60 milímetros, con una dosificación de cemento de 250 a $280 \mathrm{~kg} / \mathrm{m}^{3}$. Las resistencias exigidas debían alcanzar $200 \mathrm{kp} / \mathrm{cm}^{2}$ a los cuatro días y $300 \mathrm{kp} / \mathrm{cm}^{2}$ a los 14 , para permitir la puesta en pretensado.

\section{CALCULO DE LAS LOSAS PRETENSADAS}

\section{Generalidades}

Los métodos de cálculo desarrollados a partir de las primeras realizaciones de losas pretensadas delgadas, en 1955, están basados en la noción de carga de balance o carga equivalente de pretensado, que conseguía una

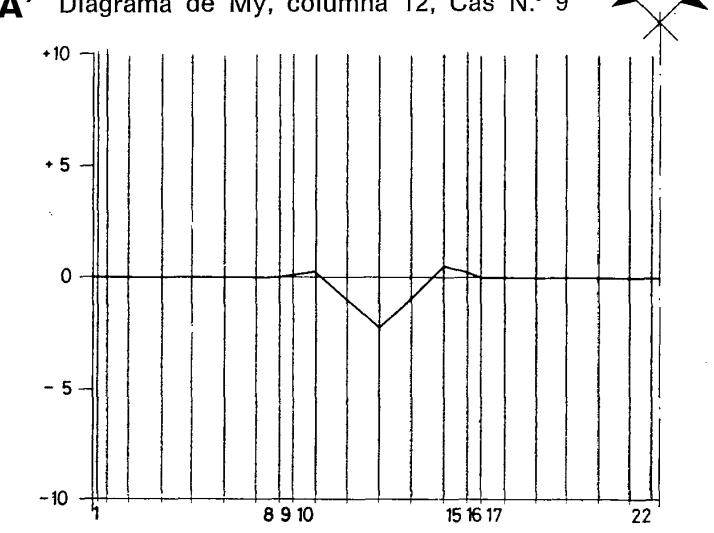

B' Diagrama de My, columna 8, Cas $\mathrm{N} .{ }^{\circ}$

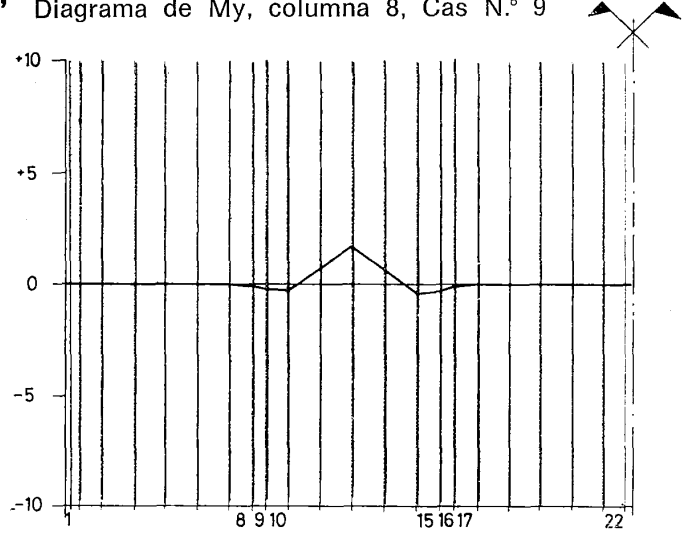

6'

Variación de $\mathrm{Mx}$, columna 12, Cas $\mathrm{N}: 9$

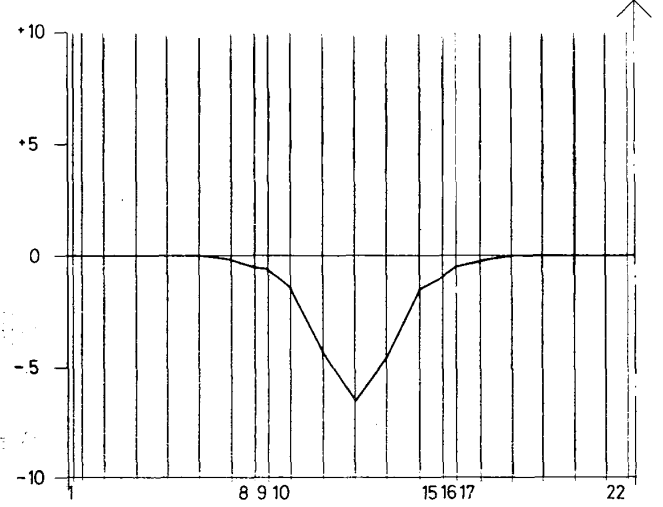

D' Variación de $\mathrm{Mx}$, columna 8, Cas N.9

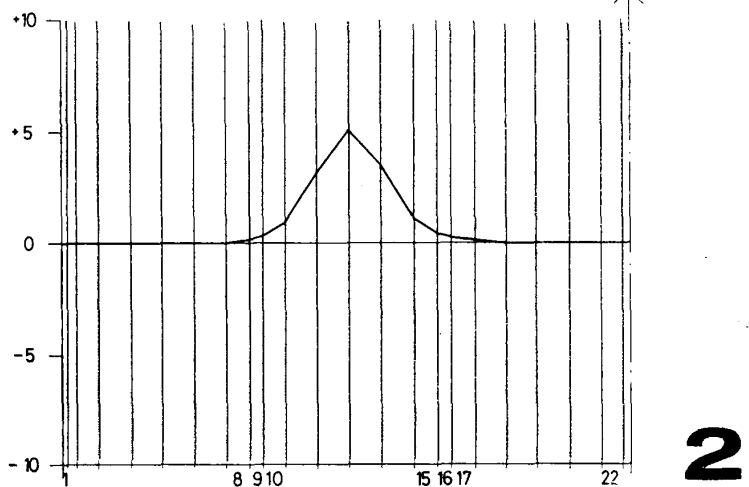


construcción sin deformaciones bajo la acción de las cargas permanentes. Estos estudios están suficientemente verificados por el gran número de ensayos llevados a cabo desde aquella fecha, lo que ha permitido formular reglas simplificadas para el dimensionamiento, aplicables a losas corrientes con luces de 7 a $10 \mathrm{~m}$, y sobrecargas uniformes de hasta unos $300 \mathrm{kp} / \mathrm{m}^{2}$. En estos casos, la disposición de los cables es un reparto no uniforme de bandas sobre los soportes y bandas centrales en las dos direcciones.

En los últimos años, diversas realizaciones suizas han utilizado otros tipos de cableado, tales como: concentración de cables sobre las líneas de apoyo en las dos direcciones (procedimiento Stahlton); y concentración de cables sobre las líneas de apoyo en una dirección, y reparto uniforme de cables en la otra. Estas disposiciones pueden conducir también a análisis simplificados, para luces y sobrecargas razonables, repartiendo los momentos totales por bandas, según métodos empíricos comparables a los desarrollados para el hormigón armado.

Las losas del centro de la Blécherette difieren de las realizaciones citadas anteriormente, por la importancia de las sobrecargas y su carácter móvil y puntual. En este caso el dimensionamiento ha debido hacerse con la ayuda de una envolvente de momentos extremos.

Por otra parte, el sistema de pendientes diseñado para favorecer el desagüe introduce, a pesar de ciertas posibilidades de inclinar los encofrados, sensibles variaciones en el espesor de la losa.

La importancia de los esfuerzos en juego se tradujo en la utilización de cables tradicionales, pero revestidos e inyectados, en vez de monocables engrasados como en las losas delgadas.

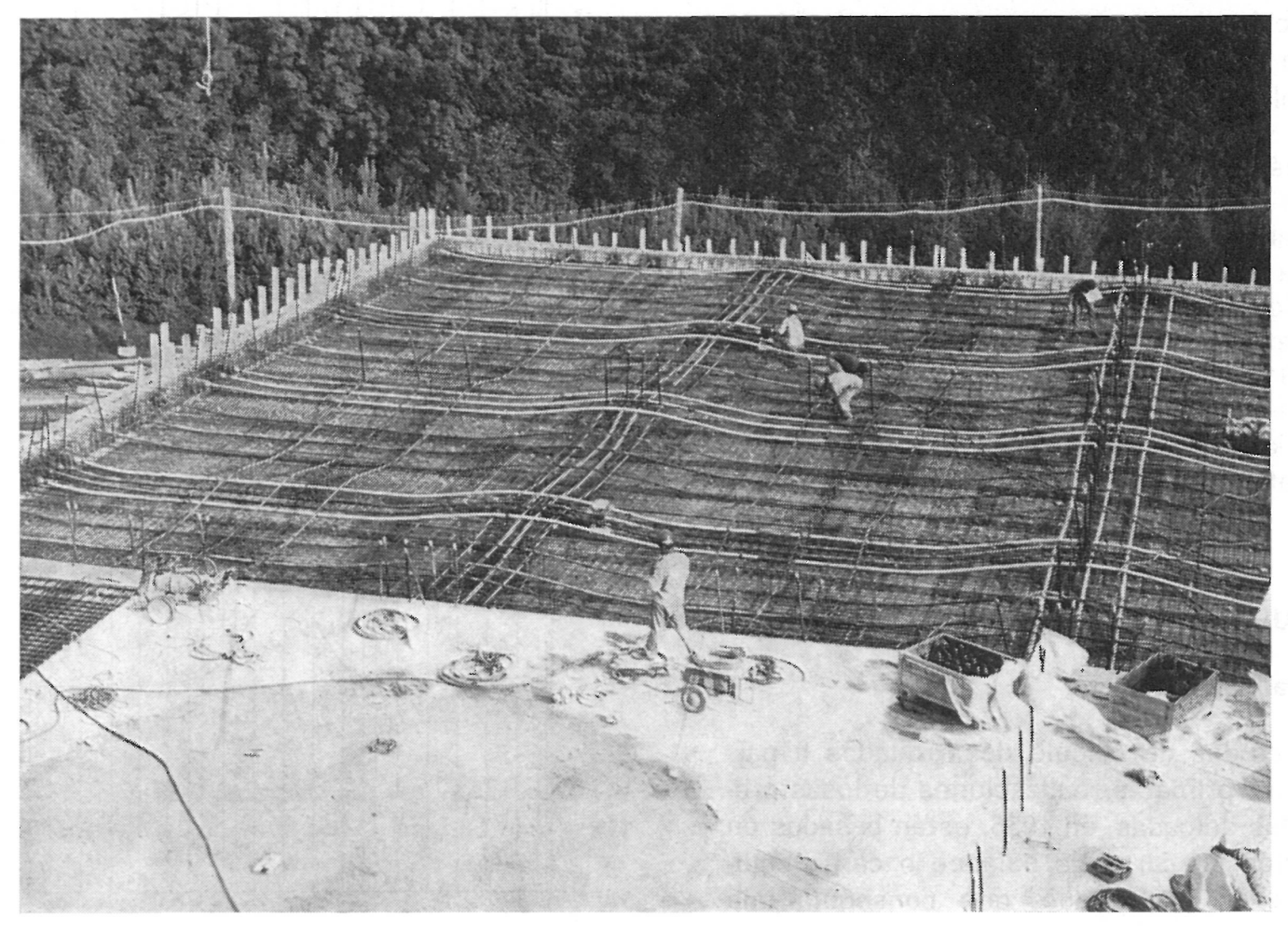




\section{CALCulo de las losas de la bléCHEREtTe}

\section{Datos básícos para el cálculo}

Los datos que han servido de base para el cálculo fueron:

- el predimensionamiento, que ha definido los valores mínimos de los espesores en los vanos y en los apoyos;

- el principio del reparto de los cables, no uniforme en las dos direcciones;

- y el estudio de las disposiciones constructivas que, a su vez, ha permitido precisar: los datos geométricos de las losas, principalmente en lo que concierne a las variaciones de espesor; las condiciones de apoyo, en particular sobre los muros; la excentricidad máxima de los cables, teniendo en cuenta las armaduras superiores e inferiores; los tipos de anclaje a utilizar en función de las etapas de ejecución; y el esquema de las armaduras, constituido por láminas de entramado superiores e inferiores, con refuerzo en los apoyos.

\section{Desarrollo del cálculo}

Los casos de cargas que se tomaron en consideración fueron: el peso propio de la losa; la sobrecarga permanente, tales como la capa de desgaste, o la tierra; el caso de carga II de la norma SIA 160 (caso determinante), y el pretensado.

El cálculo básico de los esfuerzos interiores fue hecho por medio de un programa de elementos finitos, utilizando un elemento rectangular lineal.

Para una losa de $6 \times 4 \mathrm{~m}^{2}$ de superficie fueron necesarios 70 casos de carga, para determinar los momentos de flexión y los esfuerzos cortantes debidos a las cargas permanentes y móviles, así como al pretensado. Este último fue considerado bajo forma unitaria. Los numerosos resultados obtenidos se registraron sobre una banda magnética, para su utilización posterior.

Por otra parte, el estudio manual de algunas de las secciones más solicitadas, permitió determinar los primeros coeficientes de multiplicación a aplicar en el pretensado unitario.

Los momentos de flexión del cálculo básico, los esfuerzos normales debidos al pretensado y las combinaciones de cargas móviles, constituyeron los datos de partida para el programa del cálculo del pretensado. Los datos obtenidos fueron los momentos y las compresiones normales en las fibras superiores e inferiores para los casos de esfuerzos permanentes, y esfuerzos máximos para cada dirección y los correspondientes en la otra.

El examen de estos resultados permitió ajustar los coeficientes de multiplicación del pretensado y, por aproximaciones sucesivas, tender hacia la solución que da las tracciones mínimas.

Por último, se procedió a un control del punzonamiento en las zonas que trabajan en pretensado parcial, y a una evaluación de la seguridad a la rotura.

\section{El cableado}

La distribución de los cables se caracteriza por:

- un reparto no uniforme en las dos direcciones; el $80 \%$ de la fuerza total está concentrada sobre las líneas de apoyo;

- y la colocación del cable en la posición más baja sobre la mayor longitud posible, teniendo en cuenta el radio de curvatura mínimo del mismo. 
Esta disposición, favorable por los momentos parasitarios de descarga creados en las zonas de apoyo, tiene la desventaja de provocar pérdidas por rozamiento de un 15 a un $20 \%$, según las direcciones.

\section{Cálculo básico de los esfuerzos interiores}

Para el cálculo de partida de los esfuerzos interiores se consideraron tres clases de cargas:

- la carga uniforme, compuesta por el peso propio, la sobrecarga permanente y la sobrecarga uniforme eventual;

- el pretensado, descompuesto en grupos de cables;

- la sobrecarga móvil -vagoneta de 3 ejes-.

Los grupos de cables se definieron en función de su perfil longitudinal y de la necesidad de atribuirles coeficientes de multiplicación diferenciados. La tensión nominal introducida fue de 50,100 ó 200 t, según los casos.

El efecto del cableado se simuló por la introducción de momentos aplicados a los nudos y fuerzas de desviación en los puntos donde el eje neutro presenta una rotura. Para estos esfuerzos se tuvieron en cuenta las pérdidas por rozamiento y una sobretensión momentánea de $0,75 \beta Z$, así como las etapas de ejecución.

El examen de los resultados, obtenidos principalmente bajo forma gráfica, permitió sacar conclusiones generales concernientes a cualquier grupo de cables, y así se pudo hacer converger los esfuerzos, rápidamente, hacia los valores deseados.

A título de ejemplo se dan, en la figura 2, ocho diagramas de momentos: cuatro para el caso de carga número 9 y cuatro para el caso de carga número 5 .

De los diagramas del primer caso se puede deducir que el efecto de los cables situados en los bordes no se hace sentir más que sobre el tramo donde están colocados y, que los momentos sobre el apoyo correspondientes, son despreciables.

En cuanto a la influencia de los cables del caso de carga $A$, se ve que apenas pasa de la mitad de los tramos situados de una parte a otra de los cables considerados, lo que muestra la necesidad de prever un pretensado en los bordes, para asegurar un mínimo de compresión bajo carga permanente.

El efecto de los gradientes térmicos ha sido estimado por analogía con el provocado en vigas continuas de las mismas luces, es decir: esfuerzos normales no uniformes sobre la sección; y esfuerzos de flexión, prácticamente despreciables en los tramos interiores, pero tenidos en cuenta en los de borde.

\section{Punzonamiento}

La cuestión de saber hasta qué punto el pretensado disminuye el peligro de punzonamiento no tiene, todavía, una respuesta válida.

Para las losas pretensadas de la Blécherette se realizó este cálculo sobre la base de la norma SIA número 162. En ellas, que tienen una reacción máxima de 225 t para un espesor de $46 \mathrm{~cm}$, se comprobaron las armaduras de flexión sobre el tramo y sobre los apoyos. En el segundo caso fue necesario prever una armadura superior total equivalente al $0,39 \%$ de la sección de hormigón. Esta armadura está constituida, en la sección, por: cables situados en la zona interesada, un enrejado general superior, y una cuadrícula de armaduras tradicionales. Aunque no 


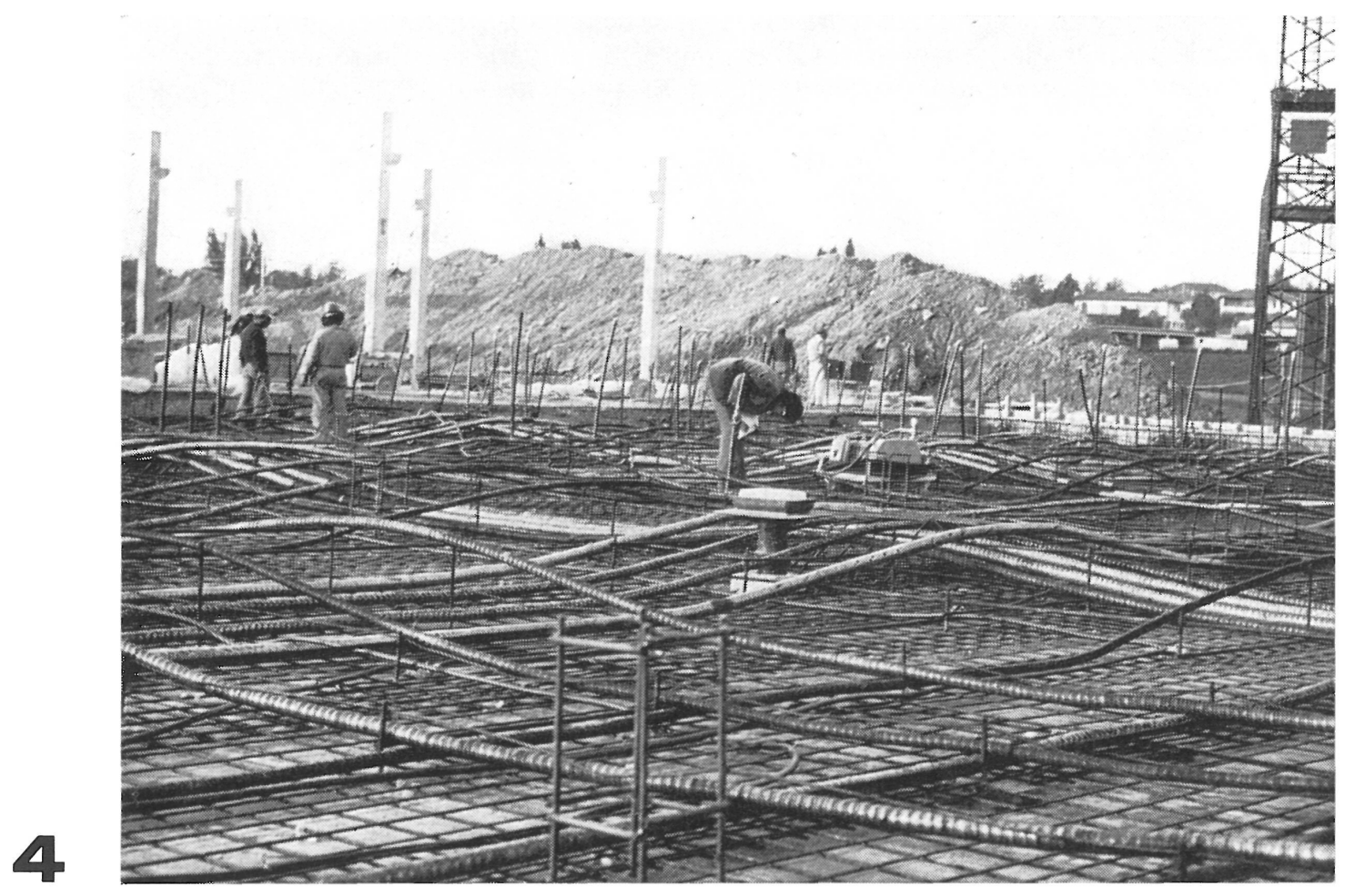

era necesario, se dispusieron algunas barras oblicuas alrededor del apoyo, para mayor seguridad.

\section{Cálculo de las armaduras}

El porcentaje mínimo de armaduras admitido fue del $0,1 \%$, de acuerdo con las recomendaciones CEB-FIP; con la excepción del $0,15 \%$ sobre los apoyos, según las normas americanas ACI para el cálculo al punzonamiento. Esto condujo a la elección de un entramado inferior uniforme del tipo Z 442.

Como ya se ha mencionado anteriormente, el control al punzonamiento determinó la sección mínima a prever sobre el apoyo.

Las zonas que trabajan en pretensado parcial bajo la acción de las sobrecargas, se comprobaron en el estado de carga II con las secciones mínimas de armaduras. El resultado fue que había que reforzar localmente la capa inferior de algunas zonas de borde.

Teniendo en cuenta los refuerzos necesarios detrás de las cabezas de anclaje, así como todas las armadras constructivas, se necesitaron, para un módulo de losa de $7,20 \times 8,40 \mathrm{~m}^{2}$ : $12,4 \mathrm{~kg} / \mathrm{m}^{2}$ de entramado de armaduras, y $3,7 \mathrm{~kg} / \mathrm{m}^{2}$ de armaduras en acero III. Esto representa un total de unos $32 \mathrm{~kg} / \mathrm{m}^{3}$.

\section{Seguridad a la rotura}

El estudio del comportamiento en el estado límite de las estructuras de losas pretensadas es bastante complejo, principalmente por la aparición de fenómenos no elásticos y de fisuración que conducen a redistribuciones de esfuerzos. Ni siquiera puede aplicarse la teoría de 
las líneas de rotura, desarrollada para las construcciones en hormigón armado, o los ensayos sobre modelos. Por ello, en este caso, el control de seguridad a la rotura se llevó a cabo de manera tradicional, considerando bandas de losas y, dentro de lo posible, el reparto de los cables.

\section{EJECUCION}

El programa de los trabajos, muy forzado, influyó notablemente en algunas disposiciones constructivas como, por ejemplo: la realización en etapas de $700 \mathrm{~m}^{2}$ de losa o de $350 \mathrm{~m}^{3}$ de hormigón colocado en un solo día; el desencofrado rápido en 4 días, después de la puesta en pretensado parcial $-60 \%$ - con el fín de permitir un ciclo de reutilización de los encofrados de dos semanas; o la puesta en tensión definitiva de los cables a los 12 ó 14 días, para permitir la colocación de los acoplamientos de la etapa siguiente o de los anclajes fijos de las juntas.

Cada etapa se desarrolló según el proceso lógico siguiente:

- Colocación y reglaje de las tablas del encofrado. Este es un modelo reforzado dada la importancia del peso muerto;

- disposición de los entramados inferiores de las armaduras de apeo y de los soportes de los cables;

- colocación de los cables de pretensado en tres capas - bandas de apoyo en un sentido, la totalidad de las del otro sentido, y las bandas centrales del primero-;

- colocación de las armaduras sobre los apoyos;

- hormigonado de las losas con la ayuda de dos grúas fácilmente desplazables, pudiendo estar dispuesta, una de ellas, sobre la losa precedente mediante apuntalamiento;

- por último, colocación de los entramados superiores para acabar de hormigonar, terminando cuidadosamente las superficies.

La precisión en la colocación de los cables se obtuvo mediante el empleo de soportes cruzados de grandes dimensiones. Estos se prefabricaron con la ayuda de un gálibo que hace intervenir las pendientes de los encofrados.

La ejecución del llaneado de la losa debía respetar las formas de las pendientes previstas para el desagüe, lo que se consiguió gracias a los caballetes de recepción de las reglas vibrantes, adaptándolos a nivel en función de su posición.

Al día siguiente al hormigonado, se sometía a la losa a una inmersión completa, facilitada por el sistema de pendientes y por el taponamiento del orificio de desagüe situado en el punto más bajo. El revestimiento con hormigón de granulometría 0-60 mm, ensayado con anterioridad, resultó totalmente satisfactorio.

\section{ENSAYO DE CARGAS}

\section{Motivo del ensayo}

Como ya se ha mencionado en párrafos anteriores, los métodos habituales para el cálculo de losas pretensadas no eran aplicables en este caso. Por tanto pareció razonable proceder a algunas pruebas sobre la obra ya realizada, con el fin de comparar los resultados con los obtenidos en el cálculo teórico. 


\section{Descripción del ensayo}

Se efectuaron dos tipos de pruebas: medida de las flechas verticales y medida de las dilataciones de la fibra superior de la losa, ambas bajo los efectos del pretensado y de dos camiones de 16 toneladas.

Las flechas se midieron mediante comparadores graduados a 1/100 de $\mathrm{mm}$. La precisión de estas medidas se puede considerar como buena.

Las dilataciones se midieron entre referencias metálicas, provistas de un agujero cónico, que habían sido fijadas previamente en las losas a distancias de $50 \mathrm{~cm}$. Para estos trabajos se utilizó un extensómetro que permitía apreciar hasta $1 / 1.000$ de $\mathrm{mm}$.

Al principio y al final de cada serie de medidas se tomó la temperatura ambiente sobre una base de acero al níquel (invar), para poder corregir las lecturas en función de las variaciones de temperatura. Sin embargo, esos efectos de la temperatura y otros factores, como los intervalos variables de tiempo entre las medidas o el número de cables puestos en tensión que provocan interacciones entre ellos mismos, son difícilmente determinables, lo que conduce a ciertos errores en las medidas.

\section{Interpretación de las medidas}

El examen de los diagramas de variación de las deformaciones muestra que el comportamiento de la losa es conforme a la lógica.

La comparación entre las flechas teóricas y las obtenidas en las pruebas demostró que el modelo matemático, utilizado para el cálculo con ordenador, fue bueno.

La comparación de los alargamientos y de los esfuerzos dieron valores bastante satisfactorios en las zonas de apoyo, mientras que en los tramos se notó alguna dispersión. Por el contrario, el valor absoluto de las dilataciones medidas es mayor sobre los apoyos, mientras que en los tramos es del mismo orden que el obtenido en los cálculos.

\section{CONCLUSIONES}

La realización del Centro de Explotación de la Blécherette comprende unos $10.500 \mathrm{~m}^{2}$ de losas sobre pilares, sirviendo de plataforma de circulación que ha permitido la puesta en obra de una estructura en hormigón pretensado que presenta las características siguientes: realiza la estanquidad, soporta las cargas de un puente de carretera y tiene deformaciones limitadas a pesar de la rapidez del desencofrado.

El estudio y la elección de los hormigones, así como el establecer un programa detallado de los trabajos han sido los principales problemas. El trabajo del ingeniero se ha revelado muy consecuente por el hecho de que las características de sobrecarga excluían, de entrada, la utilización de métodos semiempíricos.

Los cálculos han sido llevados con una cierta analogía con relación a los de un puente-losa, pero con todos los elementos bidireccionales (continuidad, pretensado, movilidad y disposición de cargas).

Gracias al ordenador y después de algunas experiencias, el dimensionamiento del pretensado y la verificación de tensados de flexión se desarrollaron normalmente. 
Por el contrario, en cuanto se aborda el control de punzonamiento de la losa y el de la seguridad a rotura, se llega a un dominio muy complejo en donde, por falta de investigación más profunda o de ensayos sobre modelos, los métodos tradicionales tienen que ser adaptados con intuición y sentido común. El desarrollo de las losas pretensadas o el interés puramente científico de la investigación permitirán quizás en el porvenir rellenar estas lagunas.

Agradecimiento a:

Propiedad:

Cantón de Vaud, representado por: Borgeaud, Mosimann y Neyroud, arquitectos SIA, Lausanne.

Chassot y Gay, ingenieros SIA, Epalinges.

Obra civil:

Asociación de Empresas: Schmalz-Sateg-Camandona, Lausanne, con la asistencia de la Oficina Técnica Piguet Ingenieros Consultores, Sociedad Anónima, Lausanne (Proyecto de Estructura).

J.-P. Cahen, arquitecto FAS/SIA, Lausanne.

Sistema de pretensado: Stahlton, S. A., Lausanne.

\section{résumé}

Les dalles précontraintes du Centre d'exploitation de la Blécherette Commune du Mont-sur-Lausanne. Suisse

Bureau Technique Piguet Ingénieurs Conseils, S. A.

La réalisation de ce Centre de la Blécherette, qui comprend environ $10.500 \mathrm{~m}^{2}$ de dalles sur poteaux, utilisées comme plateforme pour la circulation automobile, a per. béton précontraint présentant les qualités suivantes:

- elle réalise l'étanchéité,

- elle supporte les charges des ponts-

routes,

- elle subit des déformations limitées, malgré un décoffrage rapide.

$\mathrm{Si}$ les problèmes d'exécution ont surtout porté sur l'étude et le choix des bétons, ainsi que sur l'établissement d'un programme détaillé des travaux, Je travail de l'ingénieur s'est révélé très conséquent du fait des caractétistiques des surcharges qui exsemi-empiriques.

Les calculs ont été conduits avec une certaine analogie par rapport à ceux d'un pontdalle par exemple, mais avec tous les élémeints, mobilité et disposition des charges).

Grâce à l'ordinateur, et après quelque expérience, le dimensionnement de la précontrainte et la vérification des contraintes de flexion se sont deroules normalement. Par contre, dès que I'on aborde le contrôle du poinconnement de la dalle et celui de la sécurite a la rupture, on touche un domaine plus approfondies ou d'essais sur modèles, les methodes traditionnelles doivent être adoptées avec intuition et bon sens.

\section{summary}

Prestressed slabs of the Exploitation Centre at Blécherette Munícipality of Mont-sur-Lausanne - Switzerland Piguet Technical Bureau of Consulting Engineers, Ltd.

The construction of this Centre at Blécherette that consists of approximately $10,500 \mathrm{~m}^{2}$ of slabs on columns, used as a platform by the automobile traffic, has made it possible to achieve a prestressed concrete structure displaying the following characteristics:

- density,

- support of the loads of the road-bridges, tolerance of the limited deformations,
spite of a rapid forms dismtanling.

If the execution problems deal with the study and the choice of concrete, as well as with the establishment of a detailed working programme, the work of the engineer has been very related to the super-imposed use of the semi-empirical methods.

The calculations have been carried out with a certain amount of analogy in comparison those of a plate-bridge, but the bi-directional elements (continuity, prestressed, mobility and distribution
of the loads).

Thanks to the computer, and after some experimenting, the dimensioning of the prestressed parts and verification of the bending stresses developed in a normal
manner. On the other hand, the minute one enters into the control of the punching of the slab and into that of safery against breaking, one enters into a most complex research or tests on models the traditional methods have to be adapted with intuition and good common sense.

\section{zusammenfassung}

Spannbetonplatten des Ausbeutungszentrums in Blécherette Gemeinde des Mont-sur-Lausanne - Schweiz

Büro Technischer Beratender Ingenieure Piguet, S. A. Der Bau dieses Zentrums in Blécherette, das
aus ca. $10.500 \mathrm{~m}^{\circ}$ Platten auf Säulen, die als Plattform furr den Automobilverkehr die nen, besteht, hat es ermöglicht, eine Konstruktion zu gelingen, die die folgende Merk

- Dichtigkeit,

- Stützen der Last der Strassenbrücken,

- Ertragen der Deformationsgrenzen, trotz

Wenn sich die Ausführungsprobleme besonders auf das Studium und die Wahl des Betons bezichen, wie auch auf das Aufrichdoch die Arbeit des Ingenieurs, der Merkmale der Auflasten betreffend, die zu Beginn den Gebrauch der halb-empirischen Methoden ausschloss, als folgerichtig erwiesen.

Die Berechnungen sind mit gewisser Analogie- im Vergleich mit $z$. B. denen einer Elementen (Zusammenhang, Spannbeton, Beweglichkeit und Disposition der Lasten) ausgeführt worden.

Dank dem Ordner und nach einigen Experimenten, verliefen die Abmessung des Spannbetons und das Prüfen der Biegespannungen normal. Andererseits, sofort wie man das Thema der Kontrolle des Stanzens von der Platte beruihrt, sowohl als auch das der Anbruchsicherheit, kommt man gelich auf ein äussert komplexes Gebiet ein, wo auf schung und Prüfungen an Modellen, die traditionellen Methoden mit Intuition und Verstand angepasst werden müssen. 